\title{
Analysis of Victory Index at Telecommunications companies in
} Vietnam

\author{
Do Huu Hai ${ }^{1}$, Ngo Sy Trung ${ }^{2}$, Pham Van Tuan ${ }^{3} \&$ Ho Sy Ngoc ${ }^{4}$ \\ ${ }^{1}$ Information Technology Institute, Vietnam National University, Hanoi, Vietnam \\ ${ }^{2}$ Hanoi University of Home affairs, Vietnam \\ ${ }^{3}$ Faculty of Marketing, National Economics University, Vietnam \\ ${ }^{4}$ Economics Fculty of Academy of Politics Region, Vietnam \\ Correspondence: Do Huu Hai, Information Technology Institute, Vietnam National University, Hanoi, Vietnam. \\ E-mail: haidh1975@gmail.com
}

\author{
Received: September 10, 2015 Accepted: October 8, 2015 Online Published: November 20, 2015 \\ doi:10.5539/ass.v11n27p256 URL: http://dx.doi.org/10.5539/ass.v11n27p256
}

\begin{abstract}
This work is sponsored by a Vietnam National University Scientist Links (VNU - VSL project, entitled: "Analysis of Victory Index at Telecommunications companies in Vietnam" under the Grant Number QKHCN.15.0.

Currently, Vietnam telecoms market has dramatically shifted from the proprietary field to competitive one with all economic sectors, including with ones of foreign enterprises. The concentration on technology and services together with comprehensively international economic integration requires that each element of the market should have particular policies and strategies to maintain and improve market share for sustainability in the market economy. Hurwitz \& Associates' (2011) analysis methodology of victory index is used to analyze and evaluate current status, vision and development of influential corporations providing telecommunications services in Vietnam at present for giving objective assessments to consumers as well as a positive tool to providers to learn, research, maintain the competitiveness of each organization.
\end{abstract}

Keyword: Victory Index

\section{Definition}

Victory Index researched by Hurwitz and Associates (2011) is one of market research assessment instruments which are used to analyze providers on the basis of four dimensions, including Vision, Viability, Validity and Value (Hurwitz, \& Associates, 2011). The researched subject is considered as an overview about values and benefits of significant technologies. It is used to evaluate not only the engineering capability of technologies but also potentials to offer intangible values for businesses. The paper analyzes Victory Index of telecommunications companies based on data of Vietnam's ones to corroborate the conclusion of enormous potentials in the field of telecommunications.

\section{Methodologies}

For reflection on a thorough comprehension of the market and providers, the paper exploits appropriate and proper methodologies of Hurwitz and Associates together with measurements and methodologies used in the author's Doctoral thesis of "Criteria system of business culture identification - Applying for Vietnam businesses" at National Economics University, Vietnam (Hai, 2014). It is detailed clearly through the use of approximately fifty attributes of four dimensions that are calculated by a weighting algorithm. The dimensions and metrics are demonstrated and specified in Table 1.

Table 1. Description of attributes (Hurwitz \& Associates, 2011)

\begin{tabular}{ll}
\hline \multicolumn{1}{c}{ Dimensions } & \multicolumn{1}{c}{ Description } \\
\hline Vision & - Vision: transparency, practicality, a directional vision, target market conformity, judgements \\
& and roadmap for products, capacity of leadership team
\end{tabular}




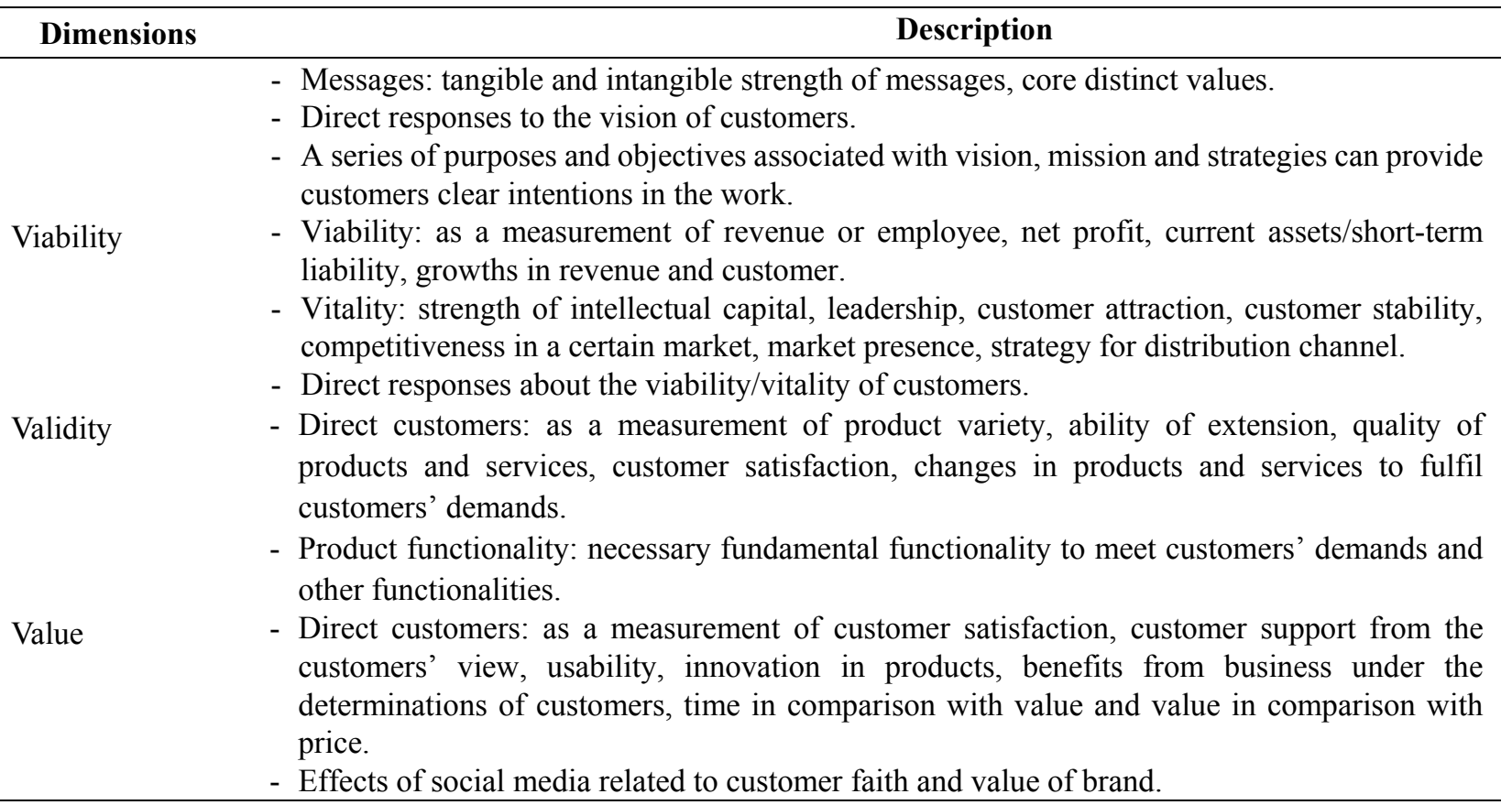

- Vision is considered as the company's strength of strategies. Evaluated attributes include whether the vision is practical and achievable in terms of time and capacity. Because building an excellent vision is not enough, companies have to draw up a well-elaborated direction that may corroborate the vision at all levels of the company. Besides, attributes consist of more well-planned aspects rather than strategies, namely including an extendable technological background, well-conveyed and influential messages and capability of positioning in the market. Other important elements are transparency, accuracy and identification of what kinds of business issues and for which customers the company must deal with. The foresight, practical experience and management skills are key elements so that leadership team can control their business operation in both adaptation to outside environment and maintain the harmony within their organization (Hai, 2014).

- Viability is considered as the company's strength as well as vitality in the market. Typically, there is no direct relationship between the company's market power and its own revenue or duration of business operation, which is particularly true in an emerging market. Sometimes, a young company can develop to be a giant one within some years if they undertake effective solutions to a complex customer issue. A complicated aggregation of financial attributes and specific ones in each company is evaluated that in case of combination, those attributes can support to identify the company's viability. This dimension also includes financial coefficient, customer attraction ratios, intellectual capital, strength of leadership team and cooperative relationships. We should also pay attention to the company's vitality in one certain market, hereby referred to telecommunications market.

- Validity is considered as strength of company's products provided to each customer. There is a difference between the product positioning and its competence in satisfying customers' requirements. Thus, Victory Index indicates the company's completion of tasks and promises. The delivered functionalities and functions are examined through the Victory Index. The Index considers the profession of functionalities and the effectiveness of a product on the basis of changes in customers' requirements. Other essential attributes include usability, innovation, capability of integrating products with other technologies and its incorporation of significant practical and theoretical standards.

- Value is regarded as advantages of products and services to customers. Even if there is an existence of a well-designed product, such product must enable to support companies to obtain their business goals. Goals range from getting the deep understanding of customers in order to achieve higher competitiveness, to applying technology to enhance outcomes. A major attribute of this dimension is how well products support businesses in accomplishing their objectives. In addition, the Value is represented through leadership's outstanding management and collection of distinct practices in management. The leadership also has clear and uniformed values to dominate the operations and set of ethical rules to make a good example for employees and competence to show their employees to distinguish what is right, what is wrong according to the author's Doctoral thesis of Criteria system of business culture indentification - Applying for Vietnam businesses (Hai, 2014). 
These dimensions are the identification of a company's strategies. The indicators of Vision and Viability are also classified into the group of "Market Strength" with these metrics, if simultaneously analyzed, present an effective expression of the strength of products and services supplies to the market. Also, if indicators of Value and Validity are examined together, they show a good illustration of customers' view on supplies. These are classified into the group of "Customer View".

\section{Analysis of Victory Index at Telecommunications Companies in Vietnam}

\subsection{Provider Selection}

The analysis illustrates that there are many different choices for customers in the market. Therefore, selected providers are not only long-term developed companies with large market share, but also emerging companies with presence and strong potential but small market share. However, all the companies are considered as the strong opponents about Victory Index.

Analyzed providers in this paper are Vietnam Posts and Telecommunications (VNPT), Military Telecommunication General Corporation (Viettel), FPT Telecom Corporation (FPT-Telecom), Vietnam Mobile Telecom Services (VMS-Mobifone), GTel Mobile Joint Stock Company (G-Tel), Saigon Posts and Telecommunications Service Corporation (SPT), Hanoi Telecom Company (Hanoi Telecom). They are regarded as leading telecommunications businesses in Vietnam, providing fixed and mobile telecommunications services, fixed and mobile broadband Internet services.

\subsection{Sources of Data}

Data are gathered from several sources: provider surveys, online surveys and other secondary data including investment reports, public documents of the government and organizations.

The data used for scoring are based on market share (the number of subscribers) to measure the scales of vision, viability, validity and value of providers of telecommunications and Internet such as mobile, fixed telephone, Internet. The data are used to analyze results in section 3.3.

\subsection{Results}

Results are calculated on the basis of a comprehensive and careful analysis of data mentioned above. Provider's scores are connected with the application of the weighting algorithm with four mentioned dimensions. Results are presented in two following charts. They are scatter plots which show the position of each provider and its own score in comparison with the average scores. Figure No. 1 (about Market Strength) is the chart of Vision versus Viability. Figure No. 2 (Customer View) is used for Validity versus Value. Some companies reach the Victory Index in both charts. They are called as double-winning companies, including Viettel and VNPT. Provider profiles are specified in the following section (Hurwitz \& Associates, 2011).

\subsubsection{Market Perspective}

Figure No. 1 shows results on the Market Perspective.

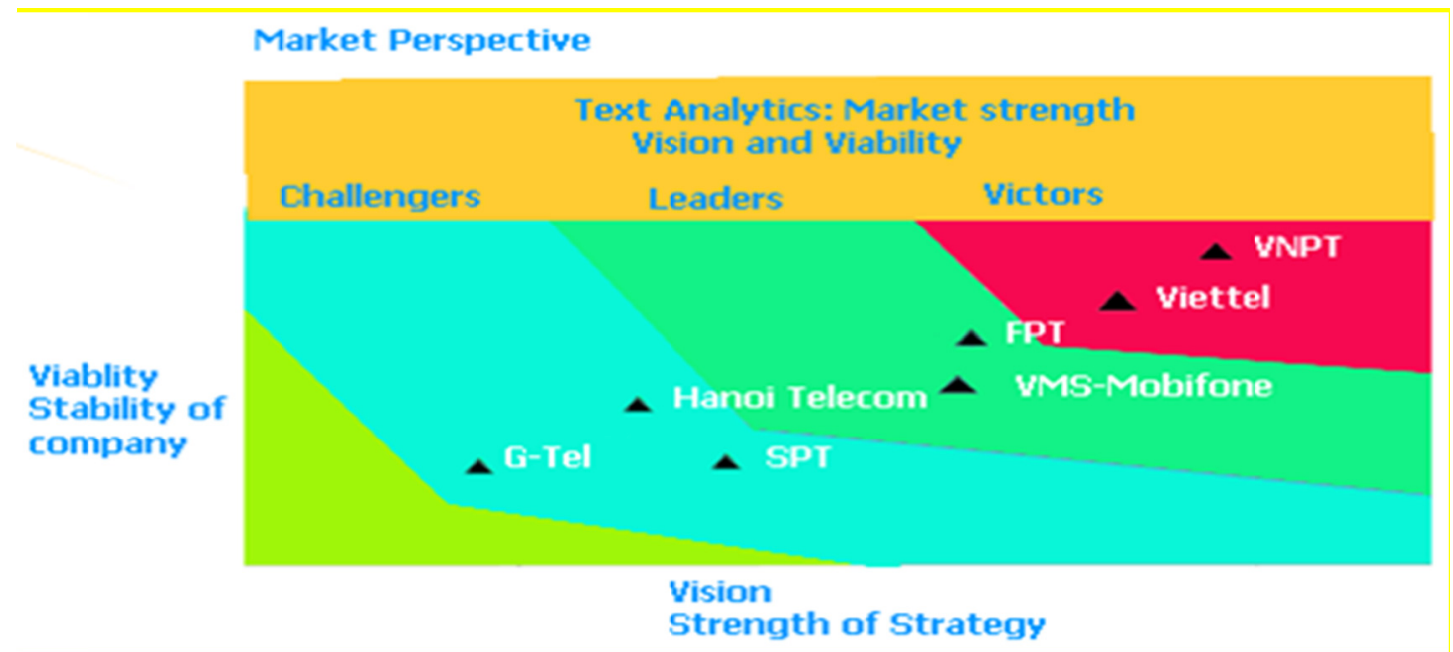

Figure 1. Results on market perspective 


\subsubsection{Victors}

The Victors show their enormous strength of brand and a solid market presence in telecommunications. They have a stable finance, a convincing vision together with successful strategies for the technology and receive customers' concurrence with their vision. Customers consider these providers are pioneers in the innovation of the telecommunications and their solutions bring about significant values. The Victor obtains the combined scores of Vision and Viability which reach to or above average scores through all of attributes calculated in both dimensions. VNPT and Viettel with their scores are regarded as Victors in the market perspective.

- Viettel is a comprehensive victor.

According to Viettel Distribution Center, Vietnam, Viettel orients itself to "become a leading innovative IT distributor in Vietnam in which IT products combined with informatics and telecommunications services is the key to our sustainable development". Respect for customers is one of core values in the business philosophy of Viettel, in which Viettel's customers are always considered separate individuals who have the needs to be cared, heard, deeply understood, best served and fairly treated in particular modes. This respect has established closeness and sympathy for consumers with the message "Understanding and maximum satisfying customers' expectations" by actual actions. Viettel's mission is "innovation to serve the people", so Viettel considers creativity as its vitality, prompt adaptation as its competitive strength and identifies the correct orientation for its development, which is consistent with the rapid change of technology and the market.

The determination of a proper vision has created immense potential for rapid and stable growth of Viettel in text analytics. Customers' satisfaction has led to steady growth in the number of subscribers of Viettel, made up the firm strength in the market as well as increased the viability of the financials. Viettel's profits have increased over years and reached 35,086 billion in 2013. Viettel has overcome VNPT to become the largest telecommunications companies in Vietnam and been ranked fifth in Vietnam Report 500 Ranking last year.

- VNPT is also a very strong victor based on the market perspective. VNPT, the oldest telecommunications company in Vietnam, are initially a State-owned company with the firm foundation and advantages of a large number fixed and mobile subscribers since the early days. The broad network and stable personnel and orientation of vision "VNPT strives for effective application of posts, telecommunications and advanced information technology to bring best values to the life of Vietnamese consumers and people" are the positive factors for the current VNPT development.

However, the powerful growth of Viettel is the reductive sign of VNPT's status. It is essentially necessary for VNPT to change to make use of their existing advantages. Firstly, for the vision and mission, VNPT cannot be "forever" the first company of telecommunications in Vietnam; therefore, the VNPT need to realize the competitive environment and improve itself in order to achieve the victory in the market. Changes in the organizational structure and strategies to increase competitiveness and market presence.

However, with the existing advantages of the first telecommunications company in Vietnam, VNPT has a large number customers, strong finance, the company is deserved as a victor on the market perspective.

\subsubsection{Leaders}

Leaders present a strong brand and stable finance and they have an excellent vision about their telecommunications solutions. However, leaders are not considered as Victors for two key reasons. Firstly, some Leaders are organizations with high viability, but they have not clearly interpreted and well implemented their vision and strategies for telecommunications as companies ranked as Victors have. Secondly, some Leaders have recently faced changes in policies of management or business, which affects directly to their performance of strategies. These companies can improve their ranking position of Victory Index if the clarity of vision is consolidated and capacity is maintained. Some of them are FPT and VMS-Mobifone.

\section{- FPT Telecom}

With its motto "All services on one connection", FPT Telecom has been constantly conducting investment, deployment and integration its increasing value-added services on one Internet connection. Investment cooperation with many large international telecommunications partners, construction of international cables, etc. are the directions in which FPT Telecom is strongly strengthening to promote its services out of Vietnam to the global market, enhancing its status of a leading telecommunications service provider. FPT Telecom is one strong company with enormous capability of conveying its own vision to customers. However, FPT-Telecom only aims at providing Internet services, so it has not dominated the market. On the basis of viability, FPT Telecom can completely develop to become a victor with more investment in this area. 
Mobifone, one of the strongly influential telecommunications companies in Vietnam, has the number of mobile subscribers about $21.40 \%$ in Vietnam. Meanwhile, Mobifone is the third largest company in Vietnamese telecommunications market and has been ranked in the $19^{\text {th }}$ in the ranking of the top 500 corporations in Vietnam).

Mobifone has undergone many changes in organizational structure with the merger and separation from VNPT, which has made the Company with permanent existing difficulties reduce competitiveness in the market. Therefore, despite being a large company with great effects on the market, Mobifone has been losing its position and needing renovations for its viability in the market. The separation of Mobifone from VNPT into a unit of the Ministry of Information and Communications is a sufficient turning point of the VMS restructuring to improve and replace the ranking positions of Vinaphone (VNPT) and Viettel.

\subsubsection{Challengers}

A Challenger can be regarded as a potential Leader or Victor; however, either it is too young in the market to be highly evaluated about its strength or there is not enough information to rank them in one of the other categories.

Be considered as strong telecommunications companies of Vietnam, the competitiveness of telecommunications companies including Hanoi Telecom, G-tel and SPT was not enough and viable in the market.

These companies with insignificant market share of fixed telecommunications and Internet services and approximately $15 \%$ of mobile subscribers in the whole nation. The companies have unclear vision and non-transparent finance. The deficiency in improving their competitiveness makes Hanoi Telecom and G-Tel risk to merge into other companies.

\subsubsection{Customer Perspective}

Figure 2, shows results on the Customer Perspective.

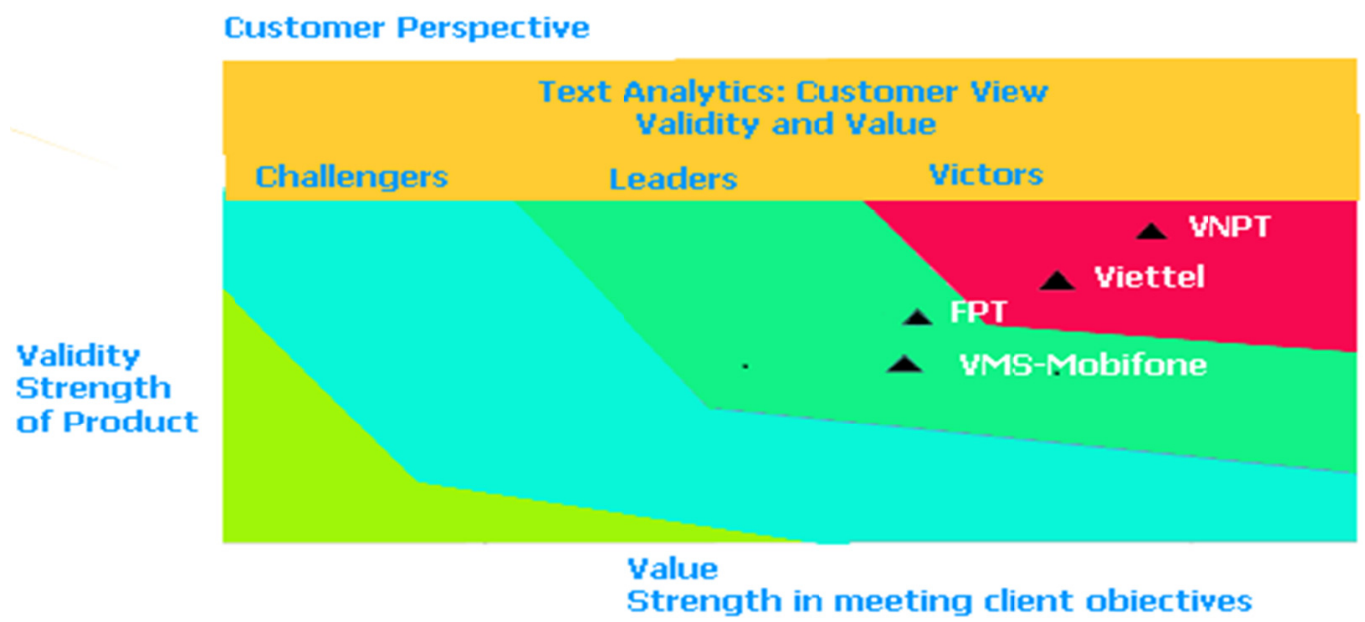

Figure 2. Results on customer perspective

\subsubsection{Victors}

The Victors illustrate admirable values of techniques and business, technology and equipment, customers' support, and holistic value as proved in customer surveys and customer interviews. Also, Victors have the remarkable depth and often width of functionality and high scores of general customer satisfaction. The combined Value and Validity scores reach at or above the average scores in this category. Viettel and VNPT are also Victors in the category of Customer perspective.

- VNPT is the Victor in the Customer perspective. As an invested telecommunications corporation with the significance of national strategies, VNPT has constantly expanded and strongly developed its telecommunications network, scale and technological innovation. Besides, VNPT has diversified services for prompt obtainment of all information as well as improvement of the life quality for the entire population nationwide; at the same time, rapidly developed services to meet the various needs of people. VNPT's investment and operation of satellites VINASAT -1 and VINASAT -2 contribute towards the perfection of national communication infrastructure and facilitate transmitting telecommunications, Internet and television services to remote, mountainous and island areas. Currently, the Next Generation Network (NGN) of VNPT is 
one of the largest network and the most modern technology in Southeast Asia. VNPT also has broadband Internet Protocol (IP) and Vietnam's largest fiber-optic backbone network used for national and international connections. Therefore, the Group has always maintained its position as leading provider of telecommunication services for many years, with the largest number of subscribers of fixed and mobile telecommunications and Internet services. This advantage makes customers consider the selection of VNPT or not. However, VNPT's first position has replaced and Viettel has become the biggest competitor of VNPT. Viettel's profits are nearly four times greater than that of VNPT in 2013. Many recommendations are given that VNPT must change and reorganize the structure to be able to regain its leading position in steading of competing with Viettel.

- Viettel emphasizes on creativity and considers prompt adaptation as its competitive strength. Strong funds and technical infrastructure promote Viettel's capability of creating and providing services for market's demands. Their products have been continuously innovated and improved in order not only to satisfy the fundamental needs of customers but also to generate the diversity and features of services. A variety of services brings about powerful combinations between mobile telecommunications and $3 \mathrm{G}$ service or between Internet and cable television. Therefore, the convenience and quality of the services are important and convincing reasons for the selection of Viettel's customers.

Viettel accounts for incessantly increasing market share in the fixed, mobile telecoms and Internet (from $12.51 \%$ to $22.96 \%$ for fixed telecoms, from $34.90 \%$ to $44.05 \%$ for mobile telecoms, from $12.57 \%$ to $29.45 \%$ for internet, from 2009 to 2013 respectively). This reveals the customer trust in Viettel. Nevertheless, there are many complaints about prices and some extra services of Viettel. In general, Viettel is fully deserved to be Victors in customer perspective.

\subsubsection{Leaders}

Leaders have powerful products as specified in scores of customer surveys. In general, customers are satisfied with their products and services and believe that such products shall bring about significant values. However, scores the obtained were lower than those of the companies at Victor status. The abstracts of the Leaders emphasize on the areas where the company got high scores and highlights some areas require their improvements.

- VMS-Mobifone is a powerful provider of mobile telecommunications in Vietnam. Mobifone passed strong growth period with high-quality services based on advanced technologies. Mobifone's status has been lower in recent years with the decreased market share. Nevertheless, VMS-Mobifone was still the third largest network in 2014 with $21.40 \%$ of subscribers, which partially reflecting that VMS-Mobifone is also a strong company in customer perspective. However, it is slowly Mobifone has been gradually losing the trust of customers in services. There is existence of several dissatisfied feedback and doubts about VMS-Mobifone. Therefore, it is extremely necessary for VMS-Mobifone to improve its apparatus and enhance quality of service and customer satisfaction.

- FPT Telecom a provider of fixed broadband Internet and value-added service on the Internet transmission as well as interactive television, hosting services, domain name registrations, etc. For fixed Internet only, there are many various sub-types of services for both consumers and enterprises. Nowadays, FPT Telecom is the second largest provider in Vietnam. Comments show that FPT-Telecom is the strongest provider in domestic transmission line; in contrast, its international transmission line is weak. In addition, Internet services often go into troubles and need maintenance. These problems are believed to be overcome when the company's infrastructure and technique become more complete.

\subsubsection{Challengers}

None of researched subjects is challenger.

\section{Conclusions}

Analysis of Victory Index at Telecommunications companies in Vietnam gives assessment of vision, viability, validity and value as well as the current status and development of influential telecommunications service providers in Vietnam. This analysis also presents a comprehensive view of values and benefits of important factors to evaluate the technique and technology, ability to offer intangible values to enterprises. From that, customers can make objective assessments, at the same time providers find out the dynamic tool for learning, researches and maintain the competitiveness of each organization.

\section{References}

Abstract retrieved from http://hurwitz.com/hurwitz-services/398-victory-index 
Hai, D. H. (2014). Criteria system of business culture indentification - Applying for Vietnam businesses (Doctoral thesis). National Economics University.

Hurwitz, \& Associates. (2011). Text Analytics: The Hurwitz Victory Index Report.

Military Telecommunication General Corporation (Viettel). Retrieved from http://viettel.com.vn/Home.html

Telecommunications - FPT Corporation. Retrieved from https://fpt.com.vn/en/business-sectors/telecommunicat ion

Vietnam Ministry of Information and Communication. (2013). Sources and statistics on Information and Communication Technology. Information and Communications Publishing House.

Vietnam Mobile Telecom Services. Retrieved from http://www.mobifone.vn/wps/portal/public

Vietnam Posts and Telecommunications. Retrieved from http://www.vnpt.vn/Default.aspx?alias=www.vnpt.vn/en

\section{Copyrights}

Copyright for this article is retained by the author(s), with first publication rights granted to the journal.

This is an open-access article distributed under the terms and conditions of the Creative Commons Attribution license (http://creativecommons.org/licenses/by/3.0/) 